\title{
Gravitational multipole moments of the Sun determined from helioseismic estimates of the internal structure and rotation
}

\author{
I. W. Roxburgh^
}

\begin{abstract}
Astronomy Unit, Queen Mary, University of London, Mile End Road, London E1 4NS, UK
LESIA, Observatoire de Paris, Place Jules Janssen, 92155 Meudon, France
\end{abstract}

Received 12 July 2001 / Accepted 31 July 2001

\begin{abstract}
We determine the gravitational multipole moments $J_{2 n}, n=1,5$, of the sun using a model of the interior structure and of solar rotation obtained from helioseismic inversions. The differential rotation of the convective zone and the underlying transition zone make only a small $(\approx 0.5 \%)$ contribution to the quadrupole moment $J_{2}$ which is found to have a value $\approx 2.21 \times 10^{-7}$.
\end{abstract}

Key words. rotation - helioseismology

\section{The external gravitational field of the Sun}

In the case of axial symmetry the external gravitational potential of the sun, $\Phi(r, \theta)$, can be expressed in the form

$\Phi=-\frac{G M}{r}\left[1-\sum_{n=2}^{\infty} J_{2 n}\left(\frac{R_{\odot}}{r}\right)^{2 n+1} P_{2 n}(\cos \theta)\right]$

where the $J_{2 n}$ are the gravitational multipole moments, $r$ the distance from the centre of the sun and $\theta$ the angle to the axis of symmetry (the rotation axis). We here determine the multipole moments using the solar internal structure and rotation determined by inverting the observed oscillation frequencies.

\section{The gravitational field inside the Sun}

The equations governing the equilibrium of a rotating star (neglecting any circulation currents) are

$\nabla P=-\rho \nabla \phi+\rho \Omega^{2} \underline{\boldsymbol{s}}$

$\nabla^{2} \phi=4 \pi G \rho$

where $P$ is the pressure, $\rho$ the density, $\phi$ the gravitational potential, $\Omega$ the angular velocity and $\underline{s}=$ $(r \sin \theta, r \cos \theta, 0)$ the vectorial distance from the rotation axis. With axial symmetry all variables are independent of $\psi$. To these equations should be added the thermal equation which determines the mean structure and any meridional circulation currents and, for equilibrium models, the angular velocity distribution (cf. Roxburgh 1964a).

* e-mail: I.W.Roxburgh@qmw.ac.uk
However for slow rotation it is sufficient to consider the effect of rotation as a small perturbation, in which case the multipole moments are determined solely by the spherically symmetric hydrostatic structure $(P(r), \rho(r), \phi(r))$ of the non rotating model, and the given angular velocity (Roxburgh 1964b). For the spherically symmetric solar structure we use the seismic model determined by Marchenkov et al. (2000) from a fully non-linear onedimensional helioseismic inversion, and for the angular velocity $\Omega(r, \theta)$ that determined by Kosovichev (1998) from an inversion using the odd splitting coefficients. For comparison we also use a standard solar reference model (Model S of Christensen-Dalsgaard et al. 1996) obtained from stellar evolution theory.

Since the ratio of centrifugal force to gravity is small we solve Eqs. (2) by perturbation analysis writing

$\rho(r, \theta)=\rho_{0}(r)+\rho_{1}(r, \theta), \quad \phi(r, \theta)=\phi_{0}(r)+\phi_{1}(r, \theta)$

where $\left|\rho_{1} / \rho_{0}\right| \ll 1, \quad\left|\phi_{1} / \phi_{0}\right| \ll 1$, with $\phi_{0}, \rho_{0}$ the values in the unperturbed, non rotating, state and $\phi_{1}, \rho_{1}$ the perturbations.

Eliminating the pressure by taking the curl (rot) of the first of Eqs. (2), and retaining only terms of first order in the perturbations gives

$\operatorname{curl}\left(\rho_{0} \nabla \phi_{1}+\rho_{1} \nabla \phi_{0}-\rho_{0} \Omega^{2} \underline{s}\right)=0$

$\nabla^{2} \phi_{1}=4 \pi G \rho_{1}$.

Eliminating $\rho_{1}$ gives the equation for the perturbation $\phi_{1}$

$\frac{1}{4 \pi G} \frac{1}{r} \frac{\mathrm{d} \phi_{0}}{\mathrm{~d} r} \frac{\partial\left(\nabla^{2} \phi_{1}\right)}{\partial \theta}-\frac{1}{r} \frac{\mathrm{d} \rho_{0}}{\mathrm{~d} r} \frac{\partial \phi_{1}}{\partial \theta}$

$=-r \frac{\partial\left(\Omega^{2} \rho_{0}\right)}{\partial r} \sin \theta \cos \theta+\rho_{0} \frac{\partial \Omega^{2}}{\partial \theta} \sin ^{2} \theta$. 
To solve this equation we expand $\phi_{1}(r, \theta)$ in terms of Legendre Polynomials $P_{2 n}(\cos \theta)$

$\phi_{1}=\phi_{10}(r)+\sum_{n=0}^{\infty} \phi_{2 n}(r) P_{2 n}(\cos \theta)$

and express the right hand side of Eq. (5) in terms of associated Legendre polynomials $P_{2 n}^{1}(\cos \theta)$

$r \frac{\partial\left(\Omega^{2} \rho_{0}\right)}{\partial r} \sin \theta \cos \theta-\rho_{0} \frac{\partial \Omega^{2}}{\partial \theta} \sin ^{2} \theta$

$=\sum_{n=2}^{\infty} A_{2 n}(r) P_{2 n}^{1}(\cos \theta)$

where the $A_{2 n}(r)$ are readily determined from the known variation of $\rho_{0}(r)$ and $\Omega(r, \theta)$ using the orthogonality of the associated Legendre polynomials. On equating coefficients of $P_{2 n}^{1}(\cos \theta)$ we obtain the set of equations for the $\phi_{2 n}(r)$

$\frac{1}{4 \pi G} \frac{1}{r} \frac{\mathrm{d} \phi_{0}}{\mathrm{~d} r}\left(\frac{1}{r^{2}} \frac{\mathrm{d}}{\mathrm{d} r}\left[r^{2} \frac{\mathrm{d} \phi_{2 n}}{\mathrm{~d} r}\right]-\frac{n(n+1) \phi_{2 n}}{r^{2}}\right)$

$-\frac{1}{r} \frac{\mathrm{d} \rho_{0}}{\mathrm{~d} r} \phi_{2 n}=-A_{2 n}(r)$.

These are subject to the boundary conditions that the $\phi_{2 n}$ are regular at $r=0$ and that at $r=R$ they match onto the external potential given in Eq. (1) which requires

$\phi_{2 n}(r) \propto r^{2 n}$ as $r \rightarrow 0$,

$r \frac{\mathrm{d} \phi_{2 n}}{\mathrm{~d} r}+\frac{(2 n+1)}{r} \phi_{2 n}=0$ at $r=R$.

Given $\rho_{0}(r), \mathrm{d} \phi_{0} / \mathrm{d} r$ from the unperturbed model, these equations can be solved to determine the $\phi_{2 n}$, and hence the multipole moments $J_{2 n}=\phi_{2 n}(R) R_{\odot} / G M_{\odot}$.

If $\Omega$ is a function of radius only, Eq. (8) reduces to that given in Roxburgh (1964b):

$\frac{1}{4 \pi G} \frac{1}{r} \frac{\mathrm{d} \phi_{0}}{\mathrm{~d} r}\left[\frac{1}{r^{2}} \frac{\mathrm{d}}{\mathrm{d} r}\left(r^{2} \frac{\mathrm{d} \phi_{2}}{\mathrm{~d} r}\right)-\frac{6 \phi_{2}}{r^{2}}\right]-\frac{1}{r} \frac{\mathrm{d} \rho_{0}}{\mathrm{~d} r} \phi_{2}$

$=\frac{1}{3} r \frac{\mathrm{d}}{\mathrm{d} r}\left(\rho_{0} \Omega^{2}\right)$.

\section{Dimensionless variables}

It is convenient to introduce the dimensionless variables

$x=\frac{r}{R_{\odot}}, \quad U=\frac{4 \pi r^{3} \rho}{M_{r}}, \quad V=\frac{\mathrm{d} \log \rho}{\mathrm{d} \log r}$,

$y_{2 n}=\phi_{2 n} \frac{R_{\odot}}{G M_{\odot}}, \omega^{2}=\Omega^{2} \frac{R_{\odot}^{3}}{G M_{\odot}}$

in terms of which Eq. (8) becomes

$\frac{1}{x^{2}} \frac{\mathrm{d}}{\mathrm{d} x}\left(x^{2} \frac{\mathrm{d} y_{2 n}}{\mathrm{~d} x}\right)-\frac{n(n+1)+U V}{x^{2}} y_{2 n}=-a_{2 n}(x)$

where the $a_{2 n}(r)$ are given by

$\left(U V \omega^{2}+U x \frac{\partial \omega^{2}}{\partial x}\right) \sin \theta \cos \theta-U \frac{\partial \omega^{2}}{\partial \theta} \sin ^{2} \theta$

$=\sum_{n=2}^{\infty} a_{2 n} P_{2 n}^{1}(\cos \theta)$.

\section{Solar structure and rotation}

Many authors have determined the internal hydrostatic structure of the sun by inversion techniques using the measured values of the solar p-mode oscillation frequencies; here we use the inverted model determined by Marchenkov et al. (2000) using the full non-linear sucessive Born approximation inversion technique. Likewise several authors have determined the solar diferential rotation from the measured rotational splitting of the frequencies; here we use the results of Kosovichev (1998) which are given in the convenient form:

$\frac{\Omega(x, \theta)}{2 \pi}=B_{1}(x)+B_{3}(x)\left(1-5 \cos ^{2} \theta\right)$

$+B_{5}(x)\left(1-14 \cos ^{2} \theta+21 \cos ^{4} \theta\right)$

where in units of $\mathrm{nHz}$ and

$B_{1}(x)=435$

$B_{1}(x)=435+51.29(x-0.71)$

$0 \leq x \leq 0.71$

$B_{1}(x)=435+882.53(1-x)$

$0.71 \leq x \leq 0.983$

$B_{3}(x)=11[1+\operatorname{erf}\{20(x-0.69)\}]$

$B_{5}(x)=-1.75[1+\operatorname{erf}\{20(x-0.69)\}]$

where $\operatorname{erf}(x)$ is the error function. The coefficients given in Kosovichev (1998) have been adjusted slightly to give $B_{1}$ continuous and $B_{3}$ the correct sign.

\section{Results}

The coefficients $a_{2 n}(x)$ were determined using this model by evaluating $\omega(x, \theta)$ and hence the left hand side of Eq. (13) on $\theta=j \pi / 12, j=1,5$, and then inverting to give the $a_{2 n}(x), n=1,5$. The resulting equations for the $y_{2 n}$ were then solved subject to the appropriate boundary conditions. The external multipole moments are given by $J_{2 n}=y_{2 n}\left(x_{\mathrm{s}}\right)$ where $x=x_{\mathrm{s}}$ is the solar surface.

Two solar models were used in the calculations:

bsun: the inverted model of Marchenkov et al. (2000).

ssun: the solar model S of Christensen-Dalsgaard et al. (1996) determined by using a stellar evolution code including diffusion, but with polynomial smoothing in the very central core $(x \leq 0.005)$.

The results are given in Table 1 .

Table 1. Solar multipole moments for $\Omega(r, \theta)$.

\begin{tabular}{|c|c|c|c|c|}
\hline model & $J_{2}$ & $J_{4}$ & $J_{6}$ & $J_{8}$ \\
\hline bsun & $2.208 \times 10^{-7}$ & $-4.46 \times 10^{-9}$ & $-2.80 \times 10^{-10}$ & $1.49 \times 10^{-11}$ \\
ssun & $2.206 \times 10^{-7}$ & $-4.44 \times 10^{-9}$ & $-2.79 \times 10^{-10}$ & $1.48 \times 10^{-11}$ \\
\hline
\end{tabular}

For comparison the value of $J_{2}$ for uniform rotation was also determined, taking the constant value to be $435 \mathrm{nHz}$ - the value given by Kosovichev for the solar 
interior. This gave the values

$$
\begin{array}{ll}
\text { bsun } & 2.220 \times 10^{-7} \\
\text { ssun } & 2.217 \times 10^{-7}
\end{array}
$$

\section{Comment on the results}

The results for the two models are in good agreement as was to be expected since there are no major differences between the inverted model and model $\mathrm{S}$ (the main differences being in the layers just below the convective zone and in the inner core). The value of $J_{2}$ for non-uniform rotation $\left(\approx 2.21 \times 10^{-7}\right)$ is close to the value obtained by Pijpers $(1998)\left(\approx 2.18 \times 10^{-7}\right)$ using a seismically determined rotation profile $\Omega(r, \theta)$, and also in close agreement with the value obtained by Paterno et al. (1996) $\left(\approx 2.15 \times 10^{-7}\right)$. They are considerably larger than the value of $\approx 5 \times 10^{-8}$ found in earlier work (Roxburgh 1964); this is to be expected since the simple model of the sun used in 1964 was much more centrally condensed that current models determined either by inversion of the observed solar oscillation frequencies, or from current evolutionary models. The value obtained is considerably larger than the value of $1.60 \times 10^{-7}$ obtained by Godier \& Rozelot (1999).

The inclusion of the differential rotation makes only a small difference $(\approx 0.5 \%)$ to the value of $J_{2}$. This is as expected. In the convective envelope and transition layer below the envelope, the departure of the rotation from its uniform value is nowhere large, the departure of the spherically averaged rotation is even smaller, and the mass and inertia of the envelope are small. We do not find the large variation by a factor of 3 in $J_{2}$ reported in Godier \& Rozelot (1999).

The value of $J_{4}$ obtained here $\approx-4.4 \times 10^{-9}$ is comparable with the values obtained by Ulrich and Hawkins (1981) using a simple theoretical model in which the rotation was assumed constant on cylinders within the convective zone and uniform in the radiative core.

We note that the value of $J_{2}$ determined here is sufficiently small so as not to play any significant role in the advance of the perihelion of Mecury, therefore supporting the prediction of the General Theory of Relativity.

\section{References}

Christensen-Dalsgaard, J., Dappen, W., Antia, H. M., et al. 1996, Science, 272, 1296

Godier, S., \& Rozelot, J.-P. 1999, A\&A, 350, 310

Kosovichev, A. G. 1998, in Sounding Solar and Stellar Interiors, IAU Symp. 181, Poster Volume, ed. J. Provost, \& F.-X. Schmider, Obs. Côte d'Azur, 97

Marchenkov, K. I., Roxburgh, I. W., \& Vorontsov, S. V. 2000, MNRAS, 312, 39

Paterno, L., Sofia, S., \& Di Mauro, M. 1996, A\&A, 314, 190

Pijpers, F. P. 1998, MNRAS, 297, 76

Roxburgh, I. W. 1964a, MNRAS, 128, 157

Roxburgh, I. W. 1964b, Icarus, 3, 92

Ulrich, R. K., \& Hawkins, G. W. 1981, ApJ, 249, 831 